\title{
Nouvelles grandeurs opérationnelles et réponse angulaire du dosimètre opérationnel
}

\author{
M. CHEMTOB*, D. FLORENCE**, G. WOLBER***
}

\author{
(Manuscrit reçu le 9 décembre 1992, \\ révisé le 20 décembre 1994, accepté le 19 janvier 1995)
}

RÉSUMÉ Les règles internationales ont défini de nouvelles grandeurs, en particulier l'équivalent de doses ambiant $H^{*}$ (surveillance des zones de travail et de l'ambiance) et $H_{\mathrm{p}}$ (surveillance individuelle, plus précisément en un point donné à l'intérieur du corps humain). Pratiquement, l'appareil de mesure ne peut être placé en ce point mais au plus près à la surface du corps. Cette différence de position induit des erreurs variables suivant la direction des rayonnements incidents et qu'il convient de corriger. La définition de nouvelles grandeurs pose le problème de l'adéquation des instruments existants et celui de la comparaison avec la dosimétrie légale par film, qui est à peu près isotrope. L'utilisation de matériel de nouvelle génération, en particulier dans les centrales nucléaires, a entraîné des écarts de mesure entre les dosimètres photographiques et le dosimètre opérationnel. Ces écarts ont entraîné une réflexion sur la réponse angulaire des dosimètres opérationnels. Cette étude traite de la variation angulaire de la grandeur opérationnelle $H_{\mathrm{p}}$. Son but est de compléter les tableaux d'un projet de norme de la Commission électrotechnique internationale (CEI) pour des valeurs d'angle comprises entre $75^{\circ}$ et $180^{\circ}$ à partir de calculs extrêmement simples qui ne sont pas en contradiction avec des études plus complètes.

ABSTRACT New quantities, especially the ambient dose equivalent $H^{*}$ and the personal dose equivalent $H_{\mathrm{p}}$ at a specified point within the body have been defined by international rules. Practically, the measurement instrument cannot be placed at this point, but at the nearest point at the surface of the body. Such a difference in position results in errors varying with the incident radiation. The definition of these new quantities raises 1) the problem of the appropriateness of the present instruments and 2) the comparison with the legal film dosemeter, which is practically isotropic. The use of new instruments, especially in nuclear power plants (NPPs), results in measurement discrepancies between photographic and operational dosemeters and thus required further work on the angular response of operational dosemeters. The angular variations of the operational quantity $H_{p}$ were considered in order to supplement the tables of an IEC draft for angle values ranging between $75^{\circ}$ and $180^{\circ}$ by means of simple calculations not contradictory with more thorough studies.

\footnotetext{
* Institut de protection et de sûreté nucléaire (IPSN), Département de protection de l'environnement et des installations (DPEI/STESR/CTHIR), BP 6, 92265 Fontenay-aux-Roses Cedex.

** EDF Production - transport, Département "Sécurité - radioprotection - environnement" (DSRE), BP 114, 93203 Saint-Denis Cedex 1.

*** EDF, Comité de radioprotection, 75384 Paris Cedex 08.
} 


\section{Grandeurs opérationnelles pour la surveillance de l'irradiation externe par les $\mathbf{X}, \gamma$ et $\beta$}

Deux groupes de grandeurs opérationnelles sont actuellement en présence pour étalonner les appareils de radioprotection effectuant une dosimétrie.

Les grandeurs opérationnelles anciennes sont :

- le kerma dans l'air ou dans les tissus $\left(K_{\mathrm{a}}\right.$ ou $\left.K_{\mathrm{t}}\right)$, toujours utilisé comme étalon primaire,

- la dose sous $3 \mathrm{~mm}$ de tissus entourés d'air $D_{\mathrm{t}}(3)$ et,

- la dose sous $0,07 \mathrm{~mm}$ de tissus entourés d'air $D_{\mathrm{t}}(0,07)$.

Ces grandeurs sont exprimées en gray et ses sous-multiples ( $\mathrm{mGy}, \mu \mathrm{Gy}$ ).

La dose $D_{\mathrm{t}}(3)$ est encore utilisée pour la surveillance de zone ou d'ambiance et du personnel pour les rayonnements $X$ et $\gamma$ d'énergie supérieure à $15 \mathrm{keV}$. La dose $D_{\mathrm{t}}(0,07)$ est encore utilisée pour la surveillance de l'irradiation par les $\mathrm{X}$ et $\gamma$ d'énergie inférieure à $15 \mathrm{keV}$ et par les $\beta$ issus des produits radioactifs. Le kerma dans l'air est utilisé pour les mesures d'ambiance en particulier dans l'environnement [2].

Les deux premières grandeurs $K_{\mathrm{a}}$ et $D_{\mathrm{t}}$ (3) sont, par définition, isotropes. La troisième $D_{\mathrm{t}}(0,07)$ est directionnelle, du fait que les rayonnements $\mathrm{X}$ et $\gamma$ de faible énergie et les $\beta$ sont directifs et diffusent peu. Cette grandeur permet une dosimétrie individuelle au niveau des doigts et du poignet ou une dosimétrie d'ambiance locale à proximité des sources émettrices. Il est implicite que l'appareil de mesure d'ambiance locale doit être orienté angulairement pour indiquer le maximum. L'appareil individuel est supposé donner une indication selon l'incidence à laquelle s'est effectuée l'irradiation sur un tissu localisé (sur le ou les doigts ou sur les poignets).

Les grandeurs opérationnelles nouvelles définies dans les documents ICRU $39,43,47$ et 51 [8-11] sont classées en deux groupes : celles relatives à la dosimétrie d'ambiance et celles réservées à la dosimétrie individuelle ; elles appartiennent à la catégorie de l'équivalent de dose et sont exprimées en sievert et ses sous-multiples (mSv et $\mu \mathrm{Sv}$ ).

Pour la dosimétrie d'ambiance, la même distinction est faite entre les rayonnements $\mathrm{X}$ et $\gamma$ d'énergie supérieure à $15 \mathrm{keV}$ et les $\mathrm{X}$ et $\gamma$ d'énergie inférieure à $15 \mathrm{keV}$, ainsi que les rayonnements $\beta$. Pour les premiers, c'est l'équivalent de dose ambiant $H^{*}(10)$ qui est la grandeur d'étalonnage. Pour les seconds, c'est l'équivalent de dose directionnel $H^{\prime}(0,07, \alpha)$ qui est la grandeur d'étalonnage. La grandeur $H^{*}(10)$ est isotrope par définition. Elle est définie dans une sphère en équivalent tissu de $30 \mathrm{~cm}$ de diamètre à $10 \mathrm{~mm}$ de profondeur sur le rayon qui fait face au faisceau considéré comme unidirectionnel et expansé. 
Il n'est pas nécessaire que l'appareil qui mesure cette grandeur soit équipé de la même sphère ICRU et, surtout, il ne faut pas l'installer sur cette sphère pour être étalonné. Il suffit que son indication, lorsqu'il est placé dans l'air, soit isotrope et égale à $H^{*}(10)$, quelle que soit l'énergie au-delà de $15 \mathrm{keV}$. En d'autres termes, la conception du dosimètre doit être telle que dans le signal de sortie du détecteur, les contributions des rayonnements directs et diffusés soient les mêmes que la sphère ICRU au point situé à $10 \mathrm{~mm}$ de profondeur face au faisceau, pour des énergies $\mathrm{X}$ et $\gamma$ supérieures à $15 \mathrm{keV}$. De ce fait, la valeur du signal reste proportionnelle à la grandeur $H^{*}(10)$.

La grandeur $H^{\prime}(0,07, \alpha)$ a les mêmes propriétés que $D_{\mathrm{t}}(0,07)$. Elle est directionnelle et explicitement anisotrope, puisque le faisceau est expansé. Elle s'applique aux ambiances locales à proximité des sources. L'appareil qui la mesure doit se comporter comme mentionné ci-dessus pour $H^{*}(10)$, c'est-àdire faire la part, dans son signal, aux rayonnements rétrodiffusés comme si la mesure s'effectuait dans la sphère à $0,07 \mathrm{~mm}$. L'opérateur doit aussi rechercher le maximum de l'indication. L'étalonnage de ces appareils est à effectuer dans l'air.

Pour la dosimétrie individuelle, deux grandeurs font la même distinction entre les $\mathrm{X}$ et $\gamma$ d'énergie supérieure à $15 \mathrm{keV}$ et les $\mathrm{X}$ et $\gamma$ d'énergie inférieure à $15 \mathrm{keV}$, ainsi que les $\beta$. Pour les premiers, c'est l'équivalent de dose personnel $H_{\mathrm{p}}(10, \alpha)$ qui est la grandeur d'étalonnage. Pour les deuxièmes, c'est l'équivalent de dose superficiel $H_{\mathrm{p}}(0,07, \alpha)$ qui est la grandeur d'étalonnage. Ces deux grandeurs individuelles diffèrent de celles définies pour l'ambiance par le fait que, pour étalonner les appareils en termes $H_{\mathrm{p}}(10, \alpha)$ et $H_{\mathrm{p}}(0,07$, $\alpha$ ), il faut installer l'appareil sur un fantôme approprié. Si le fantôme est la sphère de l'ICRU, alors :

$$
\begin{aligned}
& H_{\mathrm{p}}(10, \alpha=0)=H^{*}(10)=H^{\prime}(10, \alpha=0) \\
& H_{\mathrm{p}}(0,07, \alpha)=H^{\prime}(0,07, \alpha) \\
& \text { et encore } \\
& H_{\mathrm{p}}(10, \alpha)=H^{\prime}(10, \alpha)
\end{aligned}
$$

$H^{\prime}(10, \alpha)$ étant l'équivalent de dose directionnel sous $10 \mathrm{~mm}$ de profondeur dans la sphère ICRU.

Quand on parle de $H_{\mathrm{p}}(10, \alpha)$ et $H_{\mathrm{p}}(0,07, \alpha)$, il faut préciser le fantôme, par contre $H^{*}$ et $H^{\prime}(d, \alpha)$ sont relatifs à la sphère ICRU. Le projet de norme CEI$45 \mathrm{~B}$ (S) 104 [4] préconise, pour rendre les étalonnages plus pratiques, un fantôme "plaque" en plexiglass de dimensions $30 \mathrm{~cm} \times 30 \mathrm{~cm}$ x épaisseur $15 \mathrm{~cm}$, alors que le projet CEI 45 B (BC) 94 [3] préconisait l'utilisation de la sphère ICRU.

Comme mentionné à propos des appareils individuels mesurant $D_{\mathrm{t}}(0,07)$, les appareils mesurant $H_{\mathrm{p}}(10, \alpha)$ et $H_{\mathrm{p}}(0,07, \alpha)$ sont supposés donner une indication selon l'incidence à laquelle $s$ 'est effectuée l'irradiation sur un tissu localisé. Autrement dit : 
- une irradiation du tronc avec des rayonnements $X$ ou $\gamma$ pénétrants (énergie $>15 \mathrm{keV})$ sera surveillée en termes de $H_{\mathrm{p}}(10, \alpha)$, le fantôme servant à l'étalonnage étant le parallélépipède ;

- une irradiation des mains ou des poignets par ces mêmes rayonnements sera surveillée en termes $H_{\mathrm{p}}(10, \alpha)$; le fantôme servant à l'étalonnage doit avoir une forme simple, proche de celle des doigts, des mains ou des poignets;

- une irradiation avec des X ou des $\gamma$ mous (énergie $<15 \mathrm{keV}$ ) et $\beta$ sera surveillée en termes de $H_{\mathrm{p}}(0,07, \alpha)$; la forme du fantôme, pour l'étalonnage, dans ce cas, n'est pas critique, puisque ces rayonnements ont un faible parcours et diffusent peu.

Pour les étalonnages avec les grandeurs opérationnelles anciennes ou nouvelles d'ambiance, le dosimètre doit recevoir un faisceau parallèle et uniforme sur une surface égale ou supérieure à la surface hors tout de l'appareil. Pour les étalonnages dans les grandeurs opérationnelles individuelles $-H_{\mathrm{p}}(10, \alpha)$ et $H_{\mathrm{p}}(0,07, \alpha)$ - impliquant le fantôme, le faisceau doit être parallèle et uniforme sur la surface droite du fantôme et couvrir une surface plus grande que celle du fantôme. Dans la suite de l'étude, nous noterons la grandeur opérationnelle $H_{\mathrm{p}}(10, \alpha)$ pour être homogène avec les notations du projet CEI 45 B (BC) 94 [3]. Compte tenu du fait que le fantôme considéré est la sphère ICRU, nous aurions également pu prendre la notation $H^{\prime}(10, \alpha)$.

\section{Réponse angulaire d'un dosimètre placé sur la sphère ICRU}

\subsection{But}

Cette étude traite de la variation angulaire de la grandeur opérationnelle $H_{\mathrm{p}}(10, \alpha)$. Le projet de norme CEI 45 B (S) 120 [5] donne la variation du rapport de la lecture à alpha degrés à la lecture à $0^{\circ}$ pour des angles compris entre $0^{\circ}$ et $75^{\circ}$. Cette étude ne se veut pas normative. C'est une tentative d'explication des performances théoriques que devraient avoir des dosimètres entre $0^{\circ}$ et $180^{\circ}$. Le modèle pris est volontairement simpliste (Fig. 1).

\subsection{Modèle et hypothèses}

On ne prend en compte qu'une atténuation exponentielle et on néglige, en particulier, les effets de diffusion et d'albédo (ce qui n'aurait pas de sens pour le choix de la profondeur à laquelle on mesure, mais qui est tolérable pour expliquer le phénomène de réponse angulaire). Cette simplification peut expliquer les faibles écarts entre les valeurs des coefficients correcteurs de la norme, calculé par une méthode Monté-Carlo, et ceux trouvés par le calcul.

Nous nous sommes placés dans le cas du césium pour lequel nous avons pris un coefficient d'atténuation, $\mu$, de 0,055 . Les points où sont calculées les doses sont définis dans la figure 2 . 


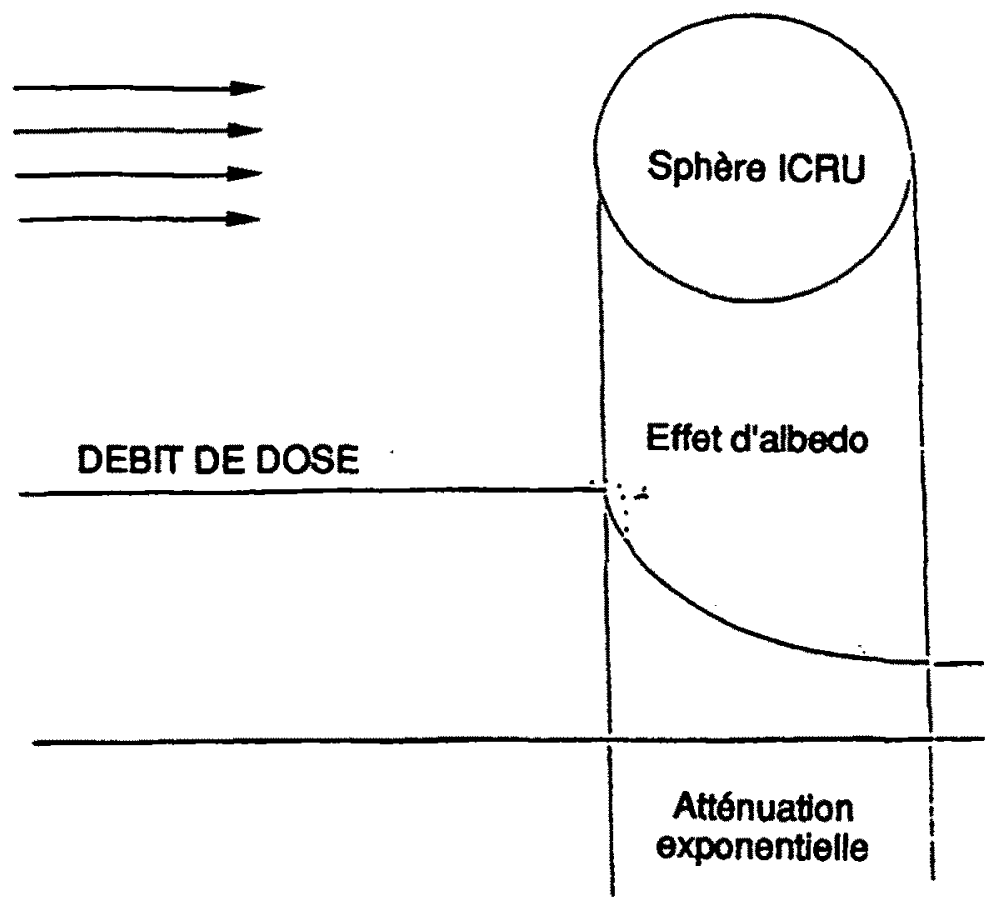

Fig. 1 - Modèle pour le calcul de la réponse angulaire d'un dosimètre.

Model for the calculation of the angular response of a dosemeter.

- soit $\mathrm{D}$ le point sur la sphère ICRU déterminant l'axe de référence,

- soit $\mathrm{H}$ un point de la sphère situé à $1 \mathrm{~cm}$ de $\mathrm{D}$ sur l'axe,

- par hypothèse la sphère ICRU est placée dans un champ expansé de direction donnée, faisant un angle $\alpha$ avec l'axe,

- soit I le point d'impact sur la sphère du rayon du faisceau passant par $\mathrm{H}$ et $\mathrm{J}$ celui correspondant à $\mathrm{D}$ (D et $\mathrm{J}$ sont confondus si $\alpha$ est compris entre 0 et 90 degrés),

- soit $d_{\mathrm{o}}$ la dose absorbée en un point du champ éloigné de la sphère.

\subsection{Etude de cas}

Nous allons étudier en fonction de l'angle $\alpha$ la variation de l'indication donnée par le dosimètre placé en $\mathrm{D}$ par rapport à la dose en $\mathrm{H}$.

\section{a) Cas général : définitions}

\section{Dose normalisée en D}

La dose au point $\mathrm{D}$ est, compte tenu de l'atténuation dans la sphère :

$d(\mathrm{D}, \alpha)=d_{\mathrm{o}} \exp -\mu \mathrm{DJ}$.

(Remarque : si $0<\alpha<90^{\circ}, \mathrm{DJ}=0$ et $d(\mathrm{D}, \alpha)=d_{\mathrm{o}}$ car on a négligé l'albedo). 


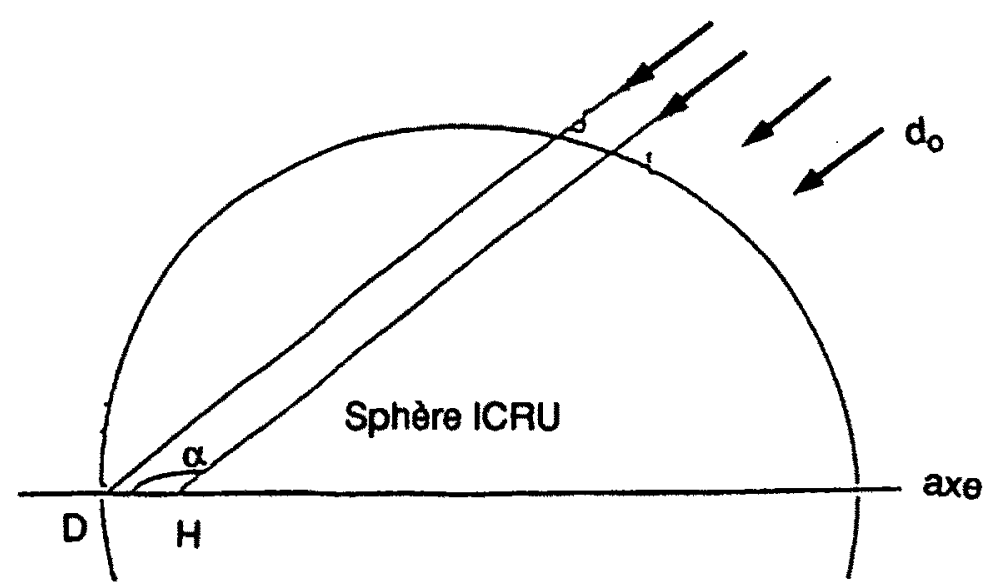

Fig. 2 - Définitions des points D, H, I, J et de l'angle d'incidence $\alpha$ pour le calcul des doses. Definitions of the points $\mathrm{D}, \mathrm{H}, \mathrm{I}, \mathrm{J}$ and of the angle of incidence $\alpha$ for dose calculations.

La dose normalisée en $\mathrm{D}$ par rapport à celle où l'angle est égal à zéro est donc :

$$
d_{\mathrm{n}}(\mathrm{D}, \alpha)=\frac{d(\mathrm{D}, \alpha)}{d(\mathrm{D}, 0)}=\frac{d(\mathrm{D}, \alpha)}{d_{0}}=\exp -\mu \mathrm{DJ}
$$

(Remarque : si $0<\alpha<90, \mathrm{DJ}=0$ et $d_{\mathrm{n}}(D, \alpha)=1$ )

\section{Dose normalisée en $\mathbf{H}$}

La dose en $\mathrm{H}$ est : $d(\mathrm{H}, \alpha)=d_{\mathrm{o}} \exp -\mu \mathrm{HI}$

La dose normalisée en $\mathrm{H}$ par rapport à celle où l'angle est égal à zéro est donc :

$$
d_{\mathrm{n}}(\mathrm{H}, \alpha)=\frac{d(\mathrm{H}, \alpha)}{d(\mathrm{H}, 0)}=\frac{\exp -\mu \mathrm{H} I}{\exp -\mu \mathrm{DH}}
$$

\section{Coefficient de variation angulaire}

Le rapport de la dose normalisée en $\mathrm{H}$ sur la dose normalisée en $\mathrm{D}$ représente la réponse angulaire que doit avoir un dosimètre placé au point $\mathrm{D}$ pour indiquer la dose au point $\mathrm{H}$ dite $H_{\mathrm{p}}(10, \alpha)$ ou $H^{\prime}(10, \alpha)$.

Nous appellerons ce ratio: "coefficient de variation angulaire" :

$$
\frac{d_{\mathrm{n}}(\mathrm{H}, \alpha)}{d_{\mathrm{n}}(\mathrm{D}, \alpha)}=\frac{(\exp -\mu \mathrm{HI}) /(\exp -\mu \mathrm{DI})}{(\exp -\mu \mathrm{DJ})}
$$

\section{b) Exemples}

Nous allons étudier en fonction de l'angle $\alpha$ les variations de la dose normalisée en $\mathrm{D}$, de la dose normalisée en $\mathrm{H}$ et du coefficient de variation angulaire. Les figures $3 a$ à $3 c$ illustrent trois exemples pour différentes valeurs $\alpha$. 
NOUVELLES GRANDEURS OPÉRATIONNELLES ET RÉPONSE ANGULAIRE

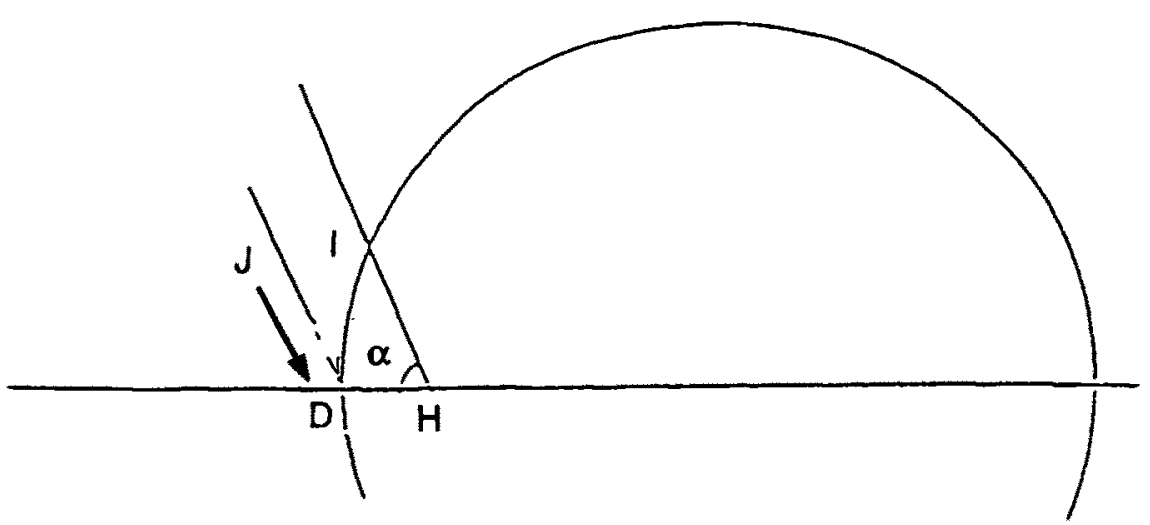

$3 a$

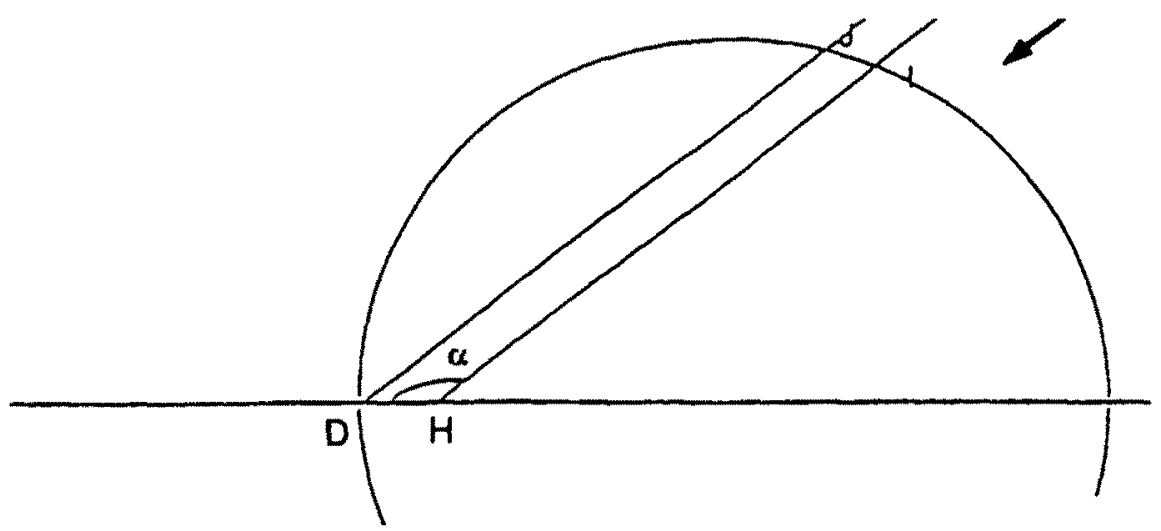

$3 b$

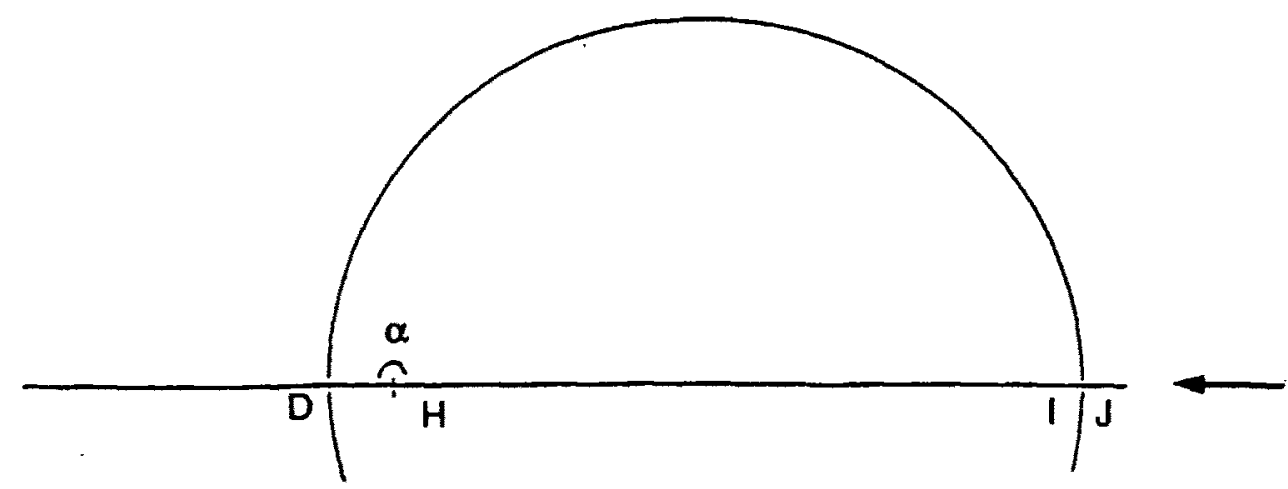

$3 c$

Fig. 3 - Variations de la dose normalisée en $\mathrm{D}$ et en $\mathrm{H}$ et du coefficient de variation angulaire (coefficient d'atténuation du césium : $\mu=0,055$ ). Exemples: a) pour $\alpha=$ $60^{\circ}$; b) pour $\alpha=120^{\circ}$; c) $\alpha=180^{\circ}$.

Variations of the normalised doses in $\mathrm{D}$ and $\mathrm{H}$ and of the angular variation coefficient. Examples : for $\alpha)=60^{\circ}$; b) $\alpha=120^{\circ}$; c) $\alpha=180^{\circ}$. 


\title{
2.4. Résultats
}

\author{
TABLEAU I
}

Dose en $H$ et réponse d'un dosimètre placé en $D$ en fonction de l'angle d'incidence

Dose at $H$ and response of a dosemeter placed at $D$ as a function of the angle of incidence

\begin{tabular}{|c|c|c|c|c|c|c|}
\hline & \multicolumn{3}{|c|}{ 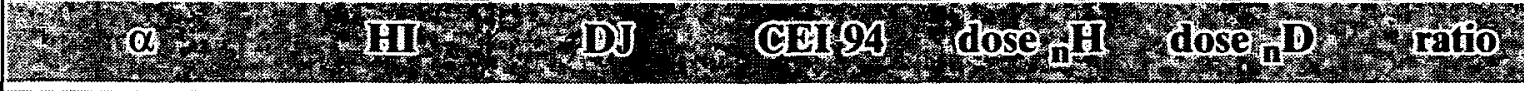 } \\
\hline 0 & 1 & 0 & 1 & 1 & 1 & 1 \\
\hline 15 & 1,0328 & 0 & 1 & 0,9982 & 1 & 0,998 \\
\hline 30 & 1,1421 & 0 & 1 & 0,9922 & 1 & 0,992 \\
\hline 45 & 1,3699 & 0 & 0,99 & 0,9799 & 1 & 0,98 \\
\hline 60 & 1,8318 & 0 & 0,96 & 0,9553 & 1 & 0,955 \\
\hline 75 & 2,8673 & 0 & 0,89 & 0,9024 & 1 & 0,902 \\
\hline 90 & 5,3852 & 0 & ND & 0,7857 & 1 & 0,786 \\
\hline 105. & 10,114 & 7,7646 & ND & 0,6058 & 0,6524 & 0,928 \\
\hline 120 & 15,832 & 15 & ND & 0,4423 & 0,4382 & 1,009 \\
\hline 135 & 21,169 & 21,213 & ND & 0,3298 & 0,3114 & 1,059 \\
\hline 150 & 25,391 & 25,981 & ND & 0,2615 & 0,2396 & 1,091 \\
\hline 165 & 28,079 & 28,978 & ND & 0,2255 & 0,2032 & 1,11 \\
\hline 180 & 29 & 30 & ND & 0,2144 & 0,192 & 1,116 \\
\hline
\end{tabular}

$\alpha$ : angle d'incidence,

$\mathrm{HI}$ : distance $\mathrm{HI}$ en $\mathrm{cm}$,

DJ : distance DJ en $\mathrm{cm}$,

CEI 94 : variation pour un rayonnement photonique du rapport de la lecture à $\alpha$ degrés à la lecture à $0^{\circ}$, pour les moniteurs utilisés pour mesurer l'équivalent de dose individuelle $H_{\mathrm{p}}$ [3], dose ${ }_{n} \mathrm{H}$ : valeur normalisée par rapport à l'angle $\alpha=0$ de l'équivalent de dose au point $\mathrm{H}$ (calculé avec une atténuation exponentielle) en fonction de l'angle $\alpha$,

dose ${ }_{\mathrm{n}} \mathrm{D}$ : valeur normalisée par rapport à l'angle $\alpha=0$ de l'équivalent de dose au point $\mathrm{D}$ (calculé avec une atténuation exponentielle) en fonction de l'angle $\alpha$,

ratio : le rapport (dose ${ }_{n} \mathrm{H} /$ dose $_{n} \mathrm{D}$ ) représente la réponse angulaire que doit avoir un dosimètre qui, une fois placé sur la sphère ICRU au point $D$, indiquera la dose au point $H$ dite $H_{\mathrm{p}}(10, \alpha)$ ou $H^{\prime}(10, \alpha)$ 
Le tableau I peut se présenter sous forme de courbes (Fig. 4)

\section{CESIUM}

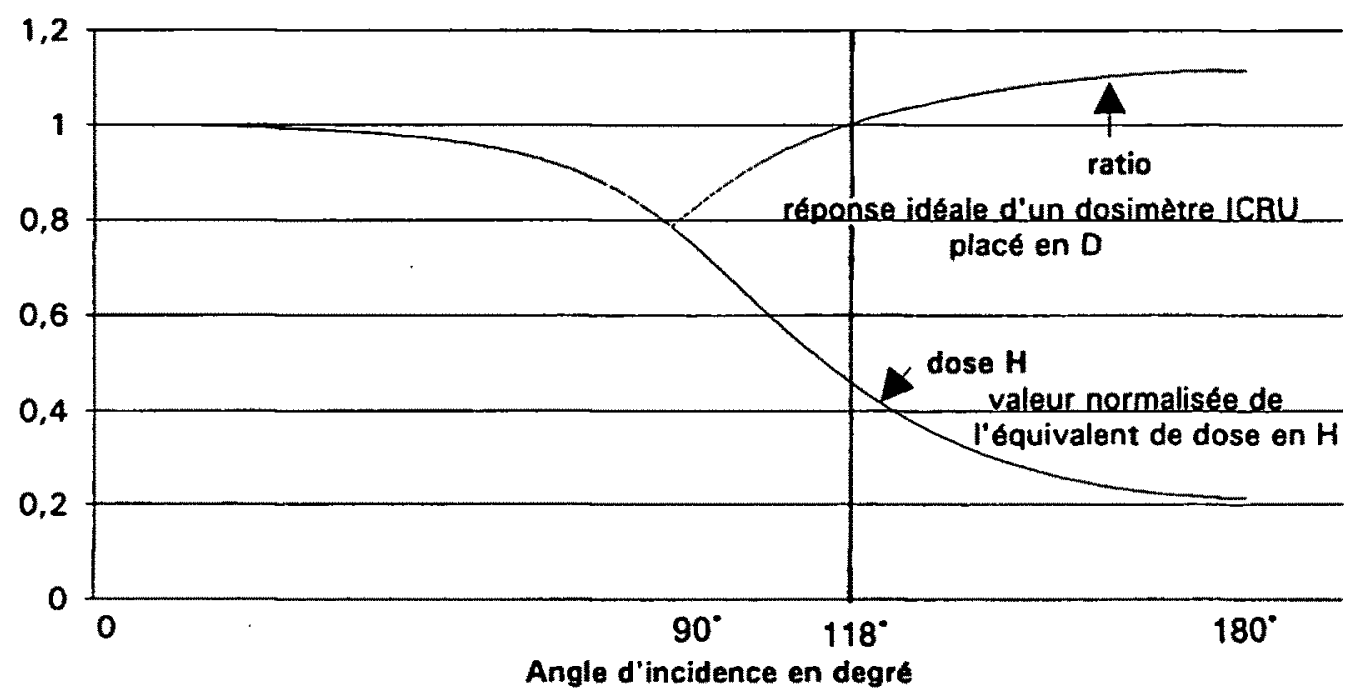

Fig. 4 - Dose en $\mathrm{H}$ et réponse d'un dosimètre placé en $\mathrm{D}$ en fonction de l'angle d'incidence. Dose at $\mathrm{H}$ and response of a dosemeter placed at $\mathrm{D}$ as a function of the angle of incidence.

\section{Commentaires sur les résultats}

\subsection{Validité de la méthode}

Pour le césium, les écarts trouvés entre les résultats calculés par une atténuation exponentielle pour l'équivalent de dose au point $\mathrm{H}$ et ceux publiés dans la littérature [7] calculés par la méthode de Monte-Carlo sont faibles (Fig. 5) :
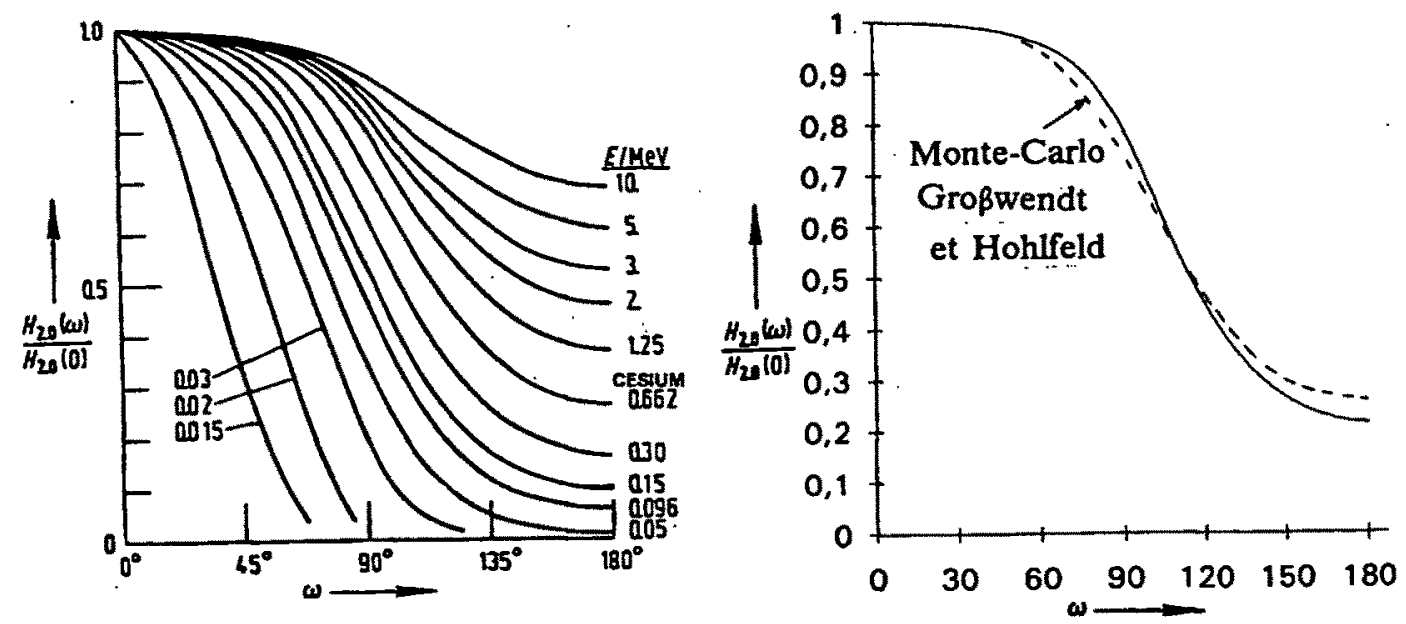

Fig. 5 - Comparaison entre les résultats calculés par la méthode de Monte Carlo et par l'atténuation exponentielle.

Comparison between the results calculated by the Monte Carlo method and by exponential attenuation. 


\subsection{Influence du rayonnement arrière sur la dose au point $\mathbf{H}$}

On considère un rayonnement isocentrique (isotrope sur un plan). Pour le césium, la part reçue par l'avant représente $70 \%$ de la dose totale reçue en $\mathrm{H}$, la part reçue par l'arrière ne représente donc que $30 \%$ (Fig. 6).

\section{CESIUM}

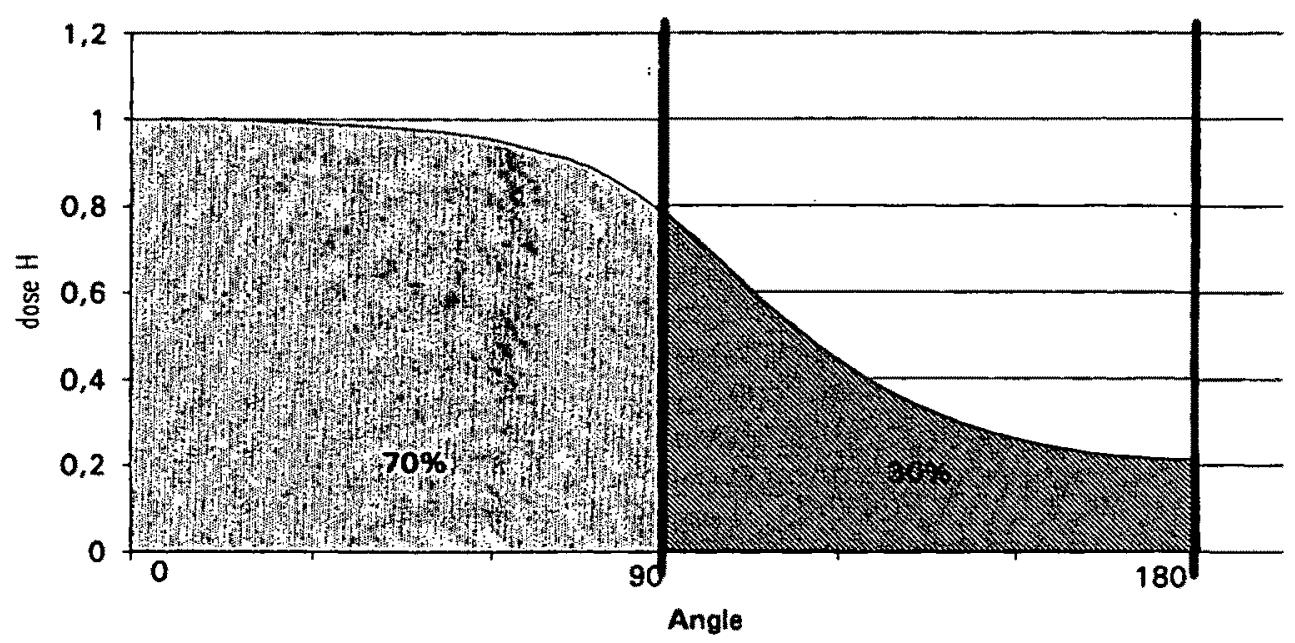

Fig. 6 - Proportions de la dose en $\mathbf{H}$ pour des incidences par l'avant et par l'arrière. Dose fractions at $\mathrm{H}$ for front and rear incidences.

\subsection{Peut-on utiliser un dosimètre isotrope?}

Pour la moitié avant de l'espace dans le cas d'un dosimètre isotrope soumis à un rayonnement isocentrique, la dose indiquée serait légèrement supérieure à la dose réelle (Fig. 7).

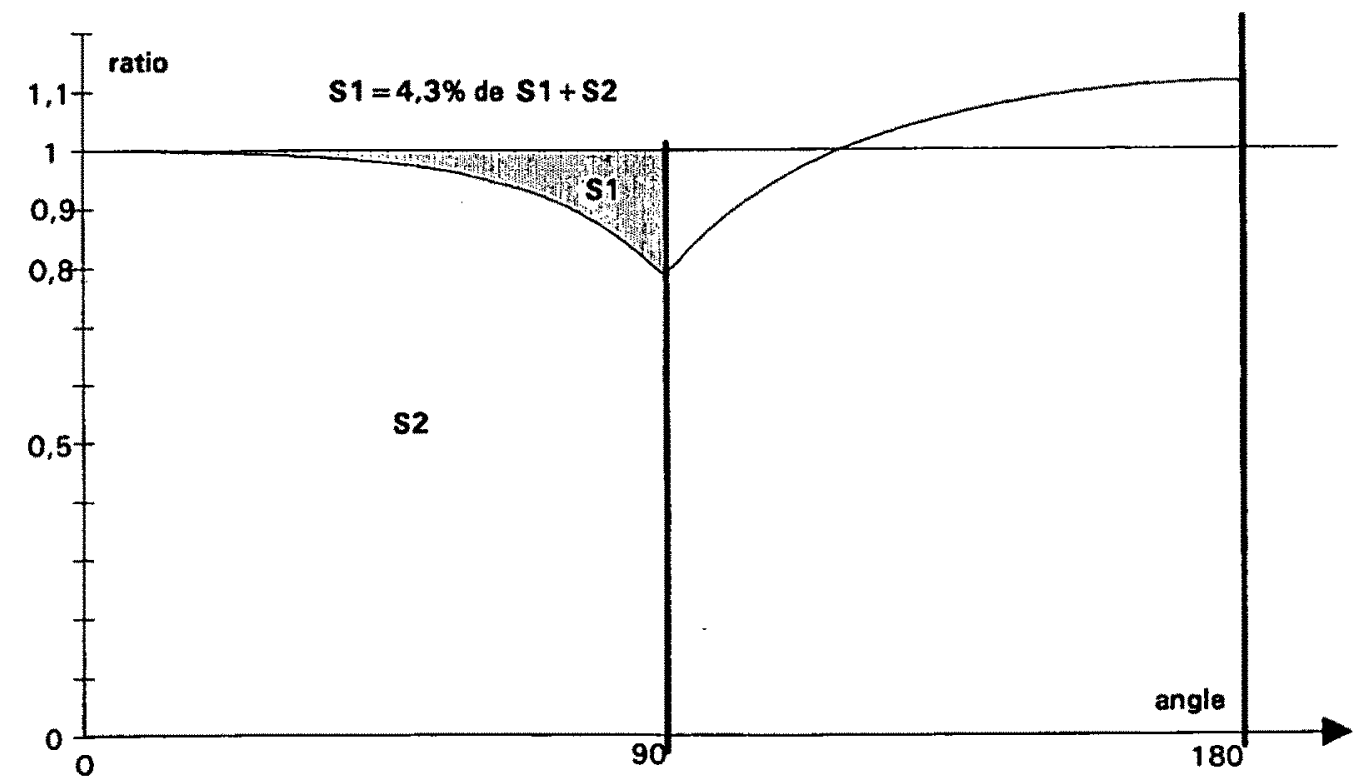

Fig. 7 - Comparaison entre la dose indiquée par un dosimètre isotrope dans l'air et par un dosimètre placé en D mesurant la dose en $\mathrm{H}$ (demi-espace avant).

Comparison between the dose indicated by an isotropical dosemeter in air and by a dosemeter placed at $\mathrm{D}$ measuring the dose at $\mathrm{H}$ (front half space). 
Pour l'ensemble de l'espace, un dosimètre isotrope placé devant la sphère ICRU et soumis à un rayonnement isocentrique indiquerait la valeur de la dose reçue (Fig. 8). Un dosimètre isotrope dans l'air est donc bien représentatif de la dose reçue par un individu en mouvement.

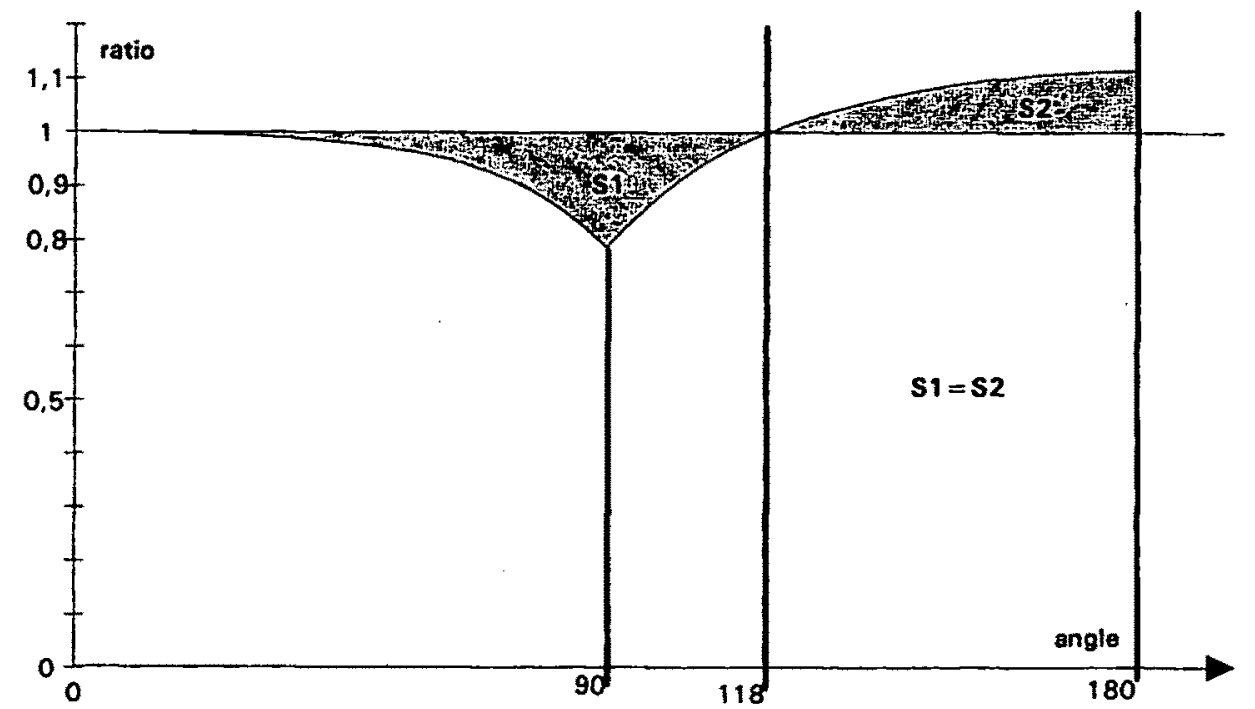

Fig. 8 - Comparaison entre la dose indiquée par un dosimètre isotrope dans l'air et par un dosimètre placé en $\mathrm{D}$ mesurant la dose en $\mathrm{H}$ (espace entier).

Comparison between the dose indicated by an isotropical dosemeter in air and by a dosemeter placed at $\mathrm{D}$ measuring the dose at $\mathrm{H}$ (total space).

\subsection{Influence de la longueur d'onde}

Pour des rayonnements peu pénétrants, la part de dose reçue par l'arrière est infime et la question de la réponse angulaire n'a pas de sens. Nous avons cependant traité le cas où $\mu=0,3$ (cas de l'américium) afin de mettre en évidence l'influence de la longueur d'onde (Fig. 9).

\section{AMERICIUM}

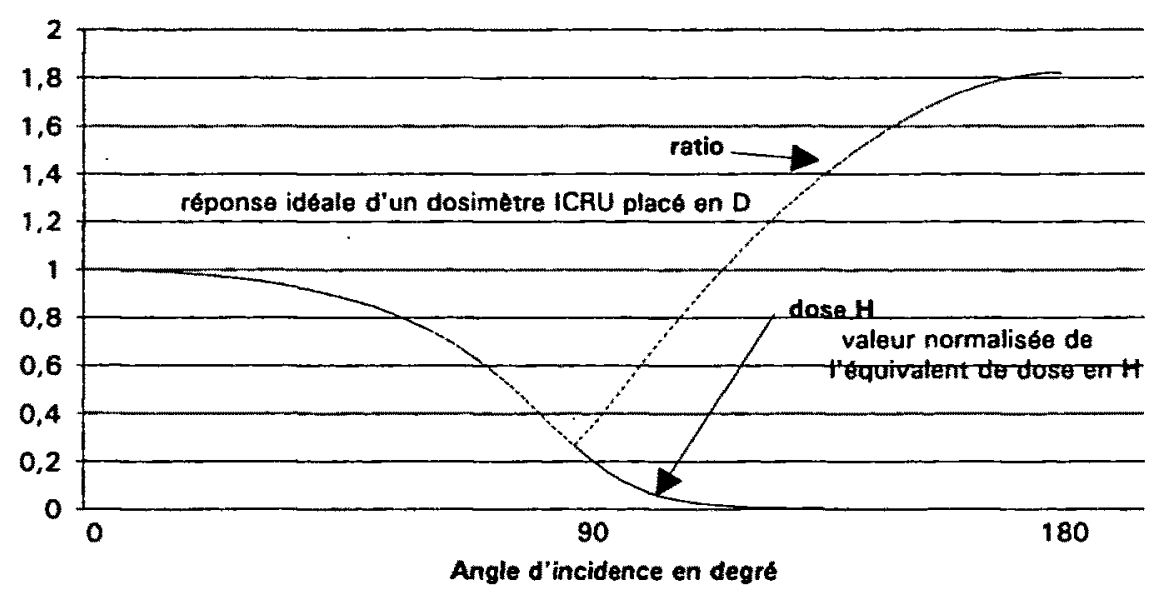

Fig. 9 - Dose en $\mathrm{H}$ et réponse d'un dosimètre placé èn $\mathrm{D}$ en fonction de l'angle d'incidence. Dose at $\mathbf{H}$ and response of a dosemeter placed at $\mathrm{D}$ as a function of the angle of incidence. 


\subsection{Cas où le coefficient angulaire est égal à 1}

Outre $\alpha=0$, cette situation correspond au cas où $\mathrm{HI}=\mathrm{DJ}+1 \mathrm{~cm}$. Cela correspond à un angle de $118^{\circ}$ qui est indépendant de la longueur d'onde de rayonnement.

\subsection{Exemple de réponse angulaire de radiamètres et de dosimètres}

Ces réponses figurent en annexe.

\section{Conclusion}

Cette étude montre, par un calcul simple, que le dosimètre à réponse isotrope satisfait à la norme CEI 45 B (BC) 94 [3] compte tenu des tolérances admises dans cette norme et pour les énergies élevées (> $660 \mathrm{keV}$ ) qui sont généralement rencontrées dans les centrales nucléaires.

\section{RÉFÉRENCES}

[1] COMMISSION ÉLECTROTECHNIQUE INTERNATIONALE (CEI) - Mesureurs d'équivalent de dose et de débit d'équivalent de dose, bêta, $X$ et gamma utilisables en radioprotection (CEI-846). Genève : CEI, 1989.

[2] COMMISSION ÉLECTROTECHNIQUE INTERNATIONALE (CEI) - Appareils portables, mobiles ou à poste fixe de mesures de rayonnement $X$ ou gamma pour la surveillance de l'environnement. I. Débitmètres (CEI-1017-1). Genève : CEI, 1991.

[3] COMMISSION ÉLECTROTECHNIQUE INTERNATIONALE (CEI) - Moniteur individuel à lecture directe d'équivalent de dose et/ou de débit d'équivalent de dose pour les rayonnements X, gamma et bêta d'énergie élevée (projet CEI-45 B (BC) 94). Genève : CEI, 1989.

[4] COMMISSION ÉLECTROTECHNIQUE INTERNATIONALE (CEI) - Moniteur individuel à lecture directe d'équivalent de dose et/ou de débit d'équivalent de dose pour les rayonnements X, gamma et bêta d'énergie élevée (projet CEI-45 B (S) 104). Genève : CEI, 1991.

[5] COMMISSION ÉLECTROTECHNIQUE INTERNATIONALE (CEI) - Moniteurs personnels à lecture directe d'équivalent de dose et/ou de débit d'équivalent de dose pour les rayonnements X, gamma et bêta d'énergie élevée et de neutrons (projet CEI-45 B (S) 120). Genève : CEI, 1993.

[6] GENCE M. DUBAIL A. - Bilan des essais entre divers dosimètres. Note interne EDF D. $581 / 77-41,1977$.

[7] GROSSWENDT B., HOHLFELD K. - Angular dependence of specified depth dose equivalent quantities in the ICRU sphere for photon radiation. Radiat. Prot. Dosim., 1982, 3, 192.

[8] INTERNATIONAL COMMISSION ON RADIATION UNITS AND MEASUREMENTS (ICRU) - Determination of dose equivalents resulting from external radiation sources (ICRU report 39). Bethesda : ICRU, 1985.

[9] INTERNATIONAL COMMISSION ON RADIATION UNITS AND MEASUREMENTS (ICRU) - Determination of dose equivalents from external radiation sources, part 2 (ICRU report 43). Bethesda : ICRU, 1988.

[10] INTERNATIONAL COMMISSION ON RADIATION UNITS AND MEASUREMENTS (ICRU) - Measurements of dose equivalents from external photon and electron radiation (ICRU report 47). Bethesda : ICRU, 1992. 
[11] INTERNATIONAL COMMISSION ON RADIATION UNITS AND MEASUREMENTS (ICRU) - Quantities and units in radiation protection dosimetry (ICRU report 51). Bethesda : ICRU, 1993.

[12] WAGNER R. - Personal dosimetry and the new quantities in radiation protection. PTBMitteilunger 98 1/88, 1988, p. 45.

[13] WOLBER G. - Grandeurs, notions et unités utilisées en radioprotection. Note interne EDF D.87/0215/6, 1987.

\section{Annexe \\ Isotropies pour différents appareils}

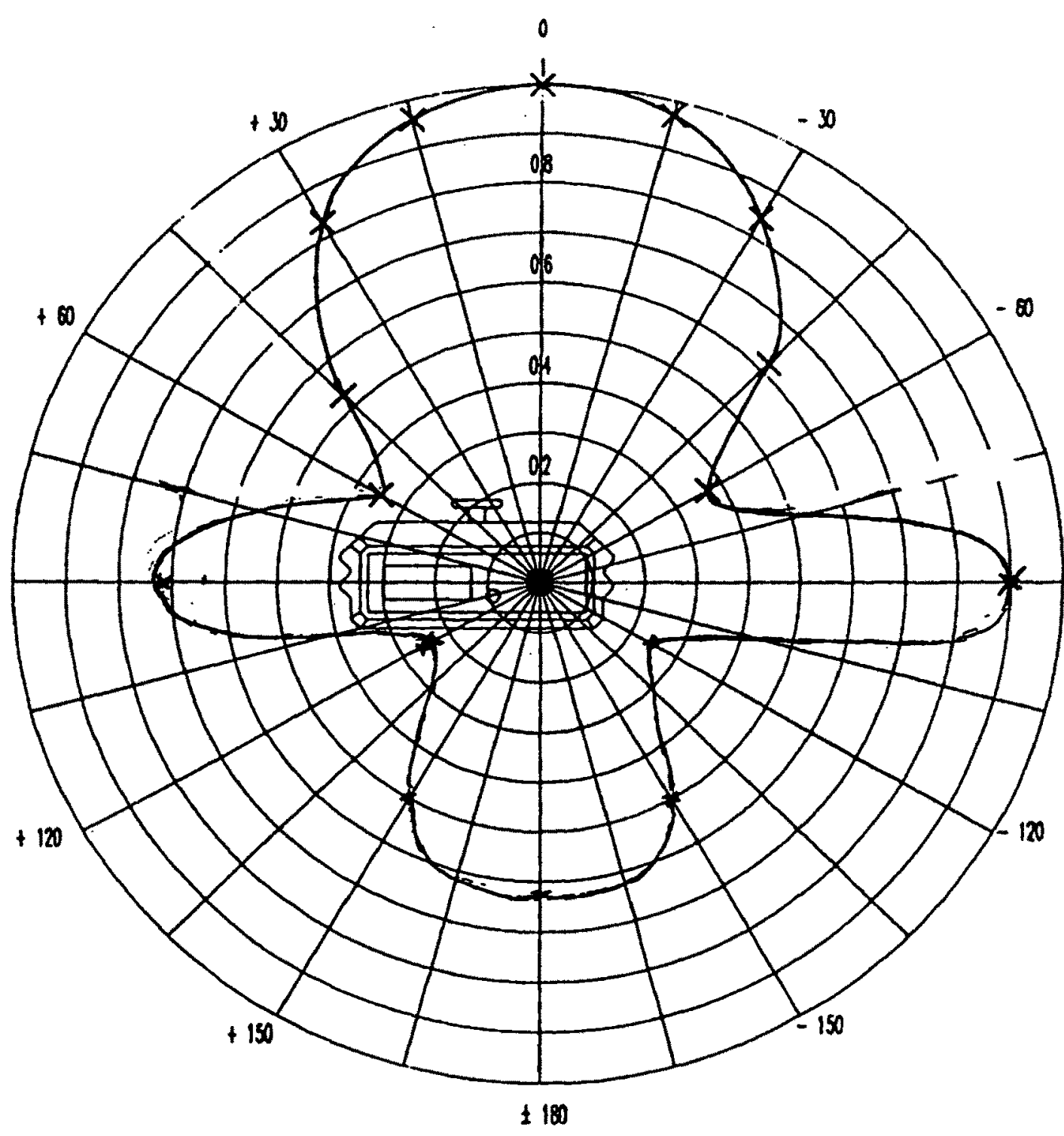

Fig. 1 - Isotropie sur le plan horizontal d'un dosimètre individuel (source : ${ }^{241} \mathrm{Am}$ ). Isotropy on the horizontal plane of a personal dosemeter (source : ${ }^{241} \mathrm{Am}$ ). 
M. CHEMTOB et al.

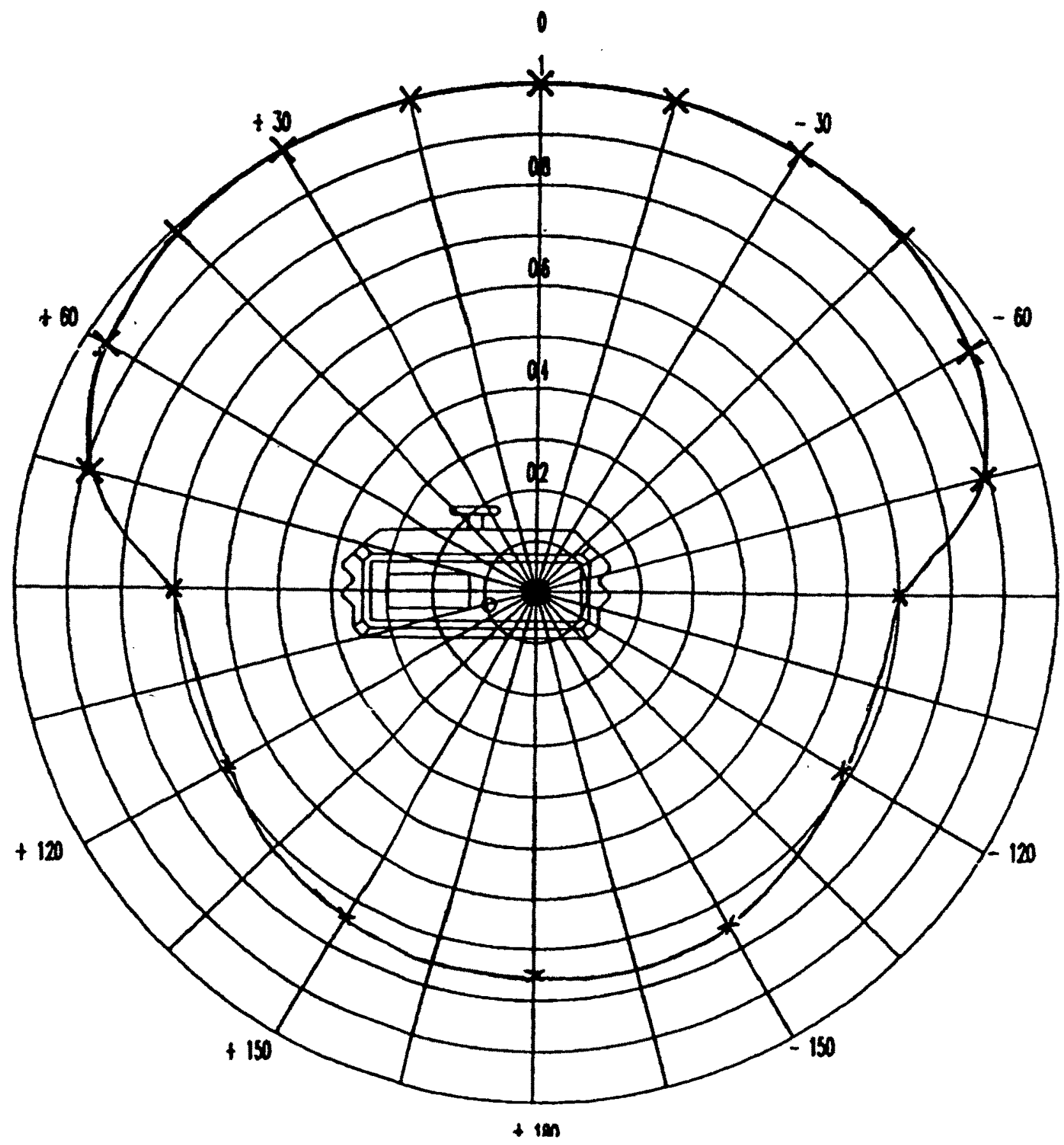

Fig. 2 - Isotropie sur le plan horizontal d'un dosimètre individuel (source: ${ }^{137} \mathrm{Cs}$ ). Isotropy on the horizontal plane of a personal dosemeter (source : ${ }^{137} \mathrm{Cs}$ ). 


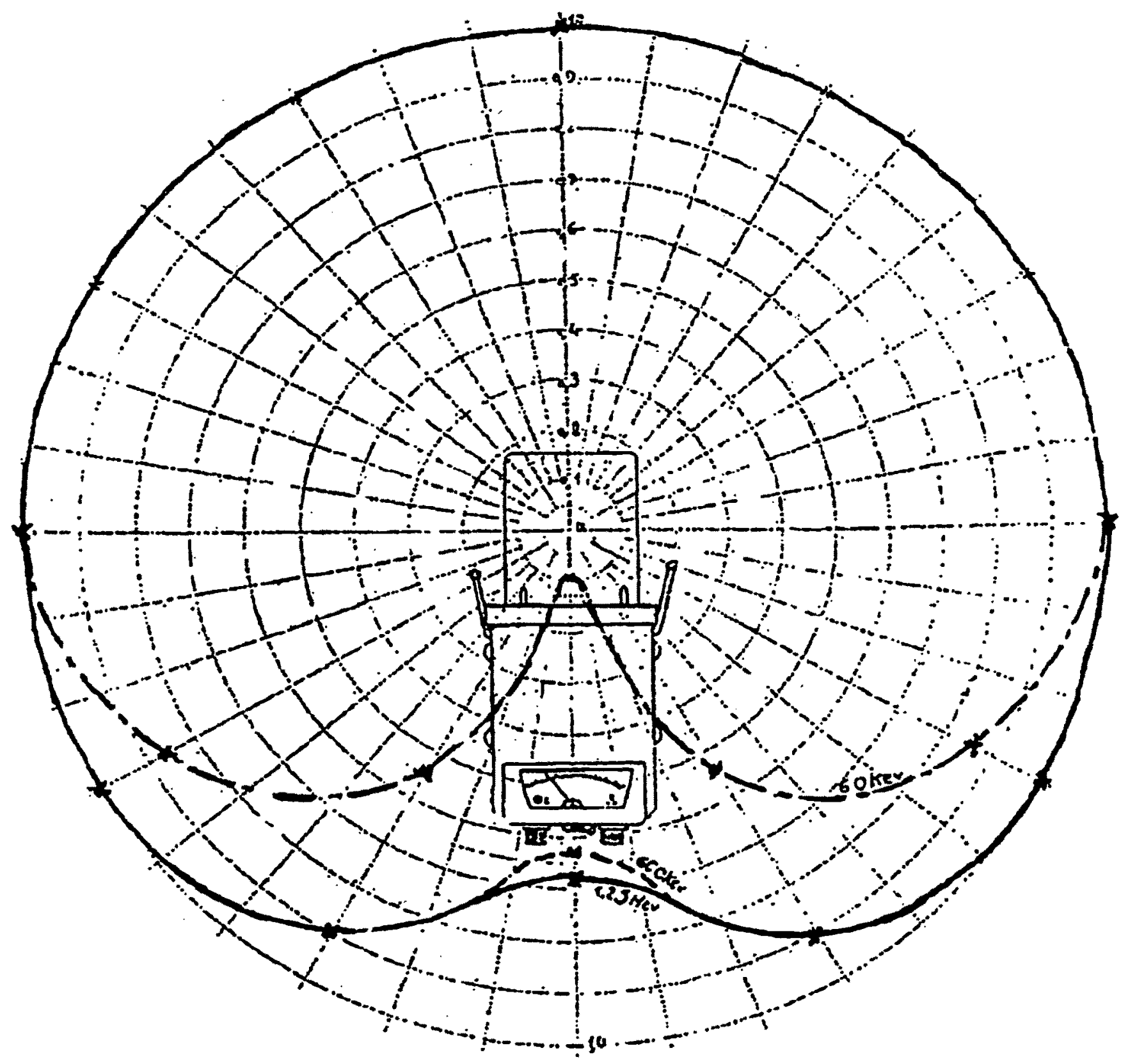

Fig. 3 - Isotropie sur le plan horizontal du dosimètre d'ambiance (Babyline). Isotropy on the horizontal plane of the ambiance dosemeter (Babyline). (一) ${ }^{60} \mathrm{Co}, 1,25 \mathrm{MeV}\left(-_{-}{ }^{137} \mathrm{Cs} 660 \mathrm{keV}(--){ }^{241} \mathrm{Am}, 60 \mathrm{keV}\right.$. 


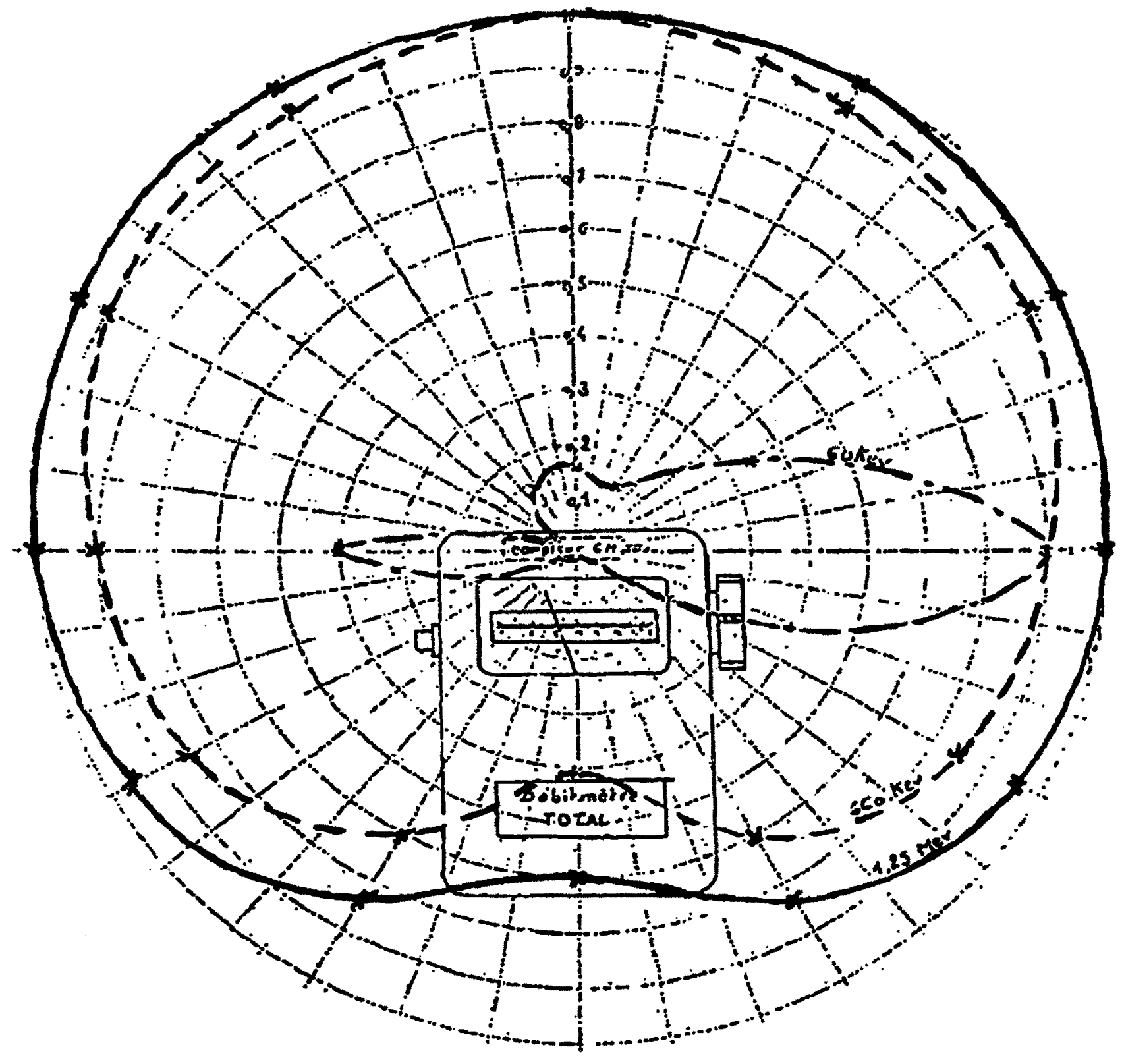

Fig. 4 - Isotropie sur le plan horizontal du dosimètre d'ambiance (Total 6150). Isotropy on the horizontal plane of the ambiance dosemeter (Total 6150). (-) ${ }^{60} \mathrm{Co}, 1,25 \mathrm{MeV}\left({ }_{-}\right){ }^{137} \mathrm{Cs} 660 \mathrm{keV} \mathrm{(-} \mathrm{-)}{ }^{241} \mathrm{Am}, 60 \mathrm{keV}$. 


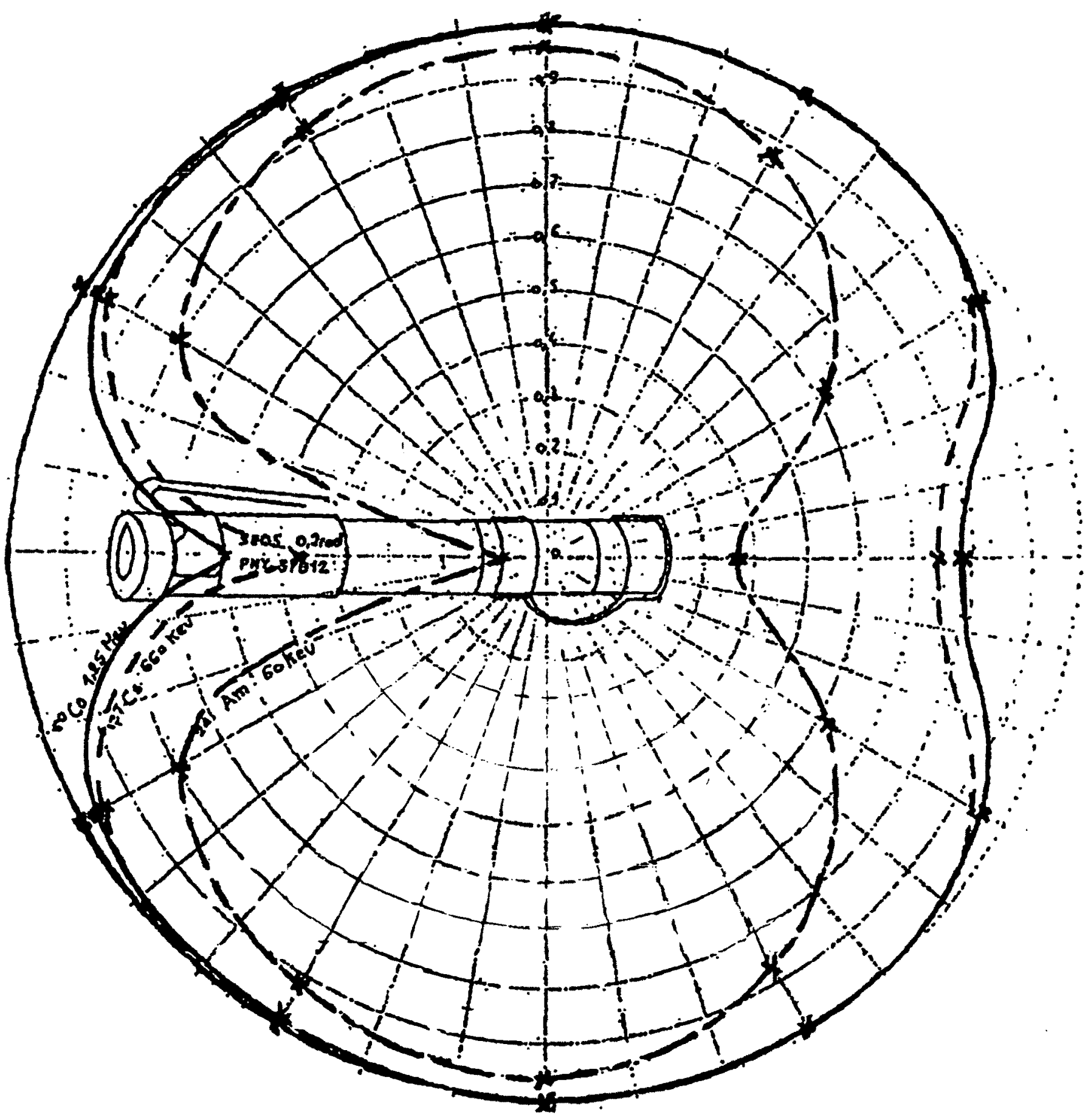

Fig. 5 - Réponse angulaire du stylo SEQ5 pour différentes énergies de rayonnement $\gamma$ situées dans un plan passant par l'axe (NB : pour un plan perpendiculaire à l'axe, le stylo est isotrope sur $2 \pi$ ).

Angular response of the pen-dosemeter, SEQ5, for various $\gamma$ energies positioned on the plane containing the axis (NB:for a plane normal to the axis, the dosemeter is isotropic on $2 \pi$ ).

(-) ${ }^{60} \mathrm{Co}, 1,25 \mathrm{MeV}(--){ }^{137} \mathrm{Cs} 660 \mathrm{keV} \mathrm{(-} \mathrm{-)}{ }^{241} \mathrm{Am}, 60 \mathrm{keV}$. 


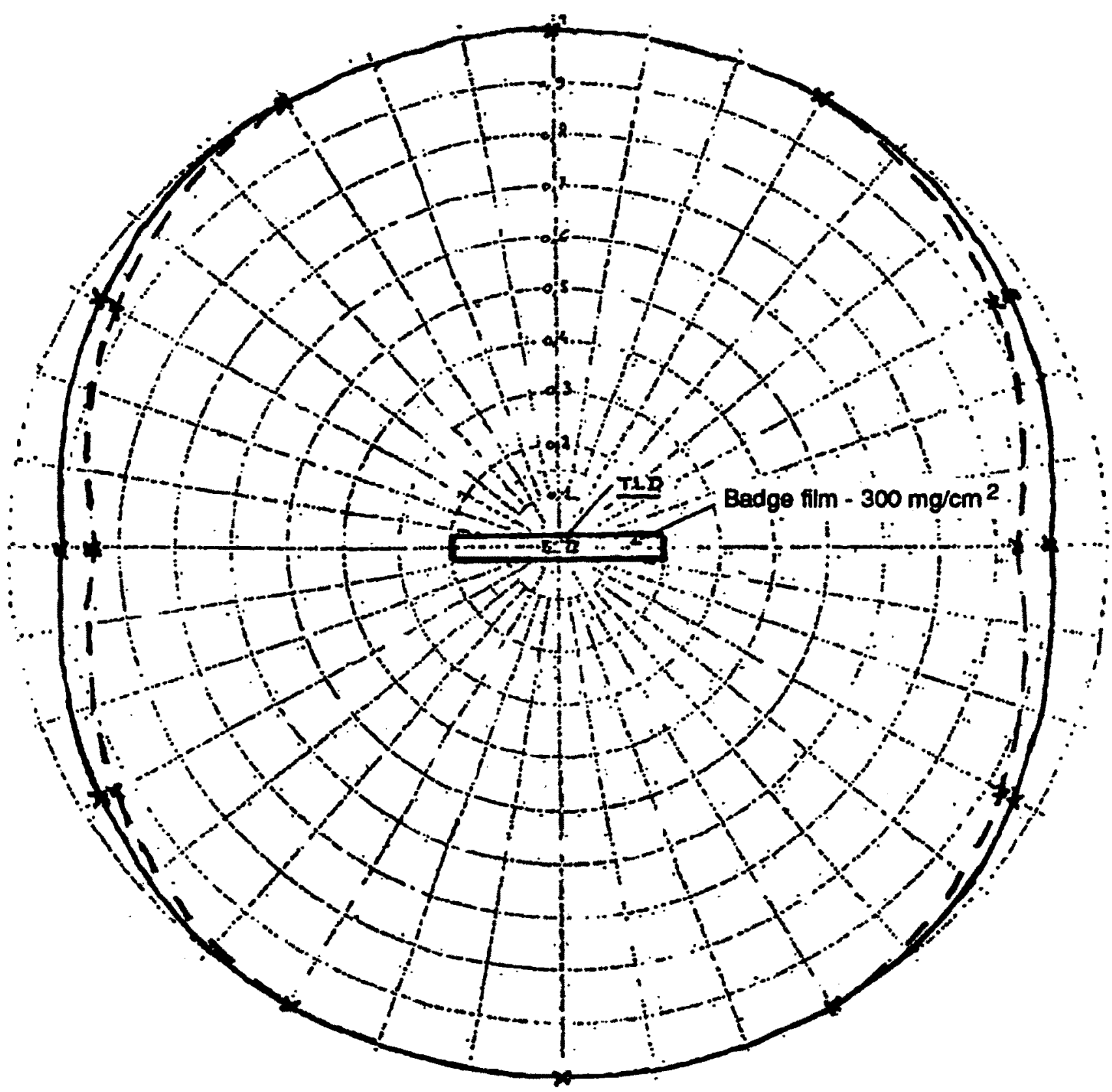

Fig. 6 - Isotropie sur le plan horizontal d'un dosimètre individuel thermoluminescent (sous $300 \mathrm{mg} \cdot \mathrm{m}^{-2}$ ).

Isotropy on the horizontal plane of a personal thermoluminescent dosemeter (under $300 \mathrm{mg} \cdot \mathrm{m}^{-2}$ ).

(一) ${ }^{60} \mathrm{Co}(---){ }^{137} \mathrm{Cs}$. 